\title{
Risk Factors of Peptic Ulcer in Military Personnel: A Systematic Review of the Literature
}

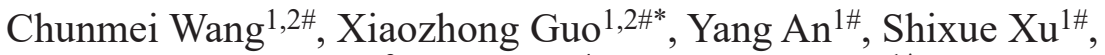 \\ Dan Zhang ${ }^{3}$, Yong Qin ${ }^{4}$ and Xingshun $\mathrm{Qi}^{{ }^{*}}$

\begin{abstract}
${ }^{1}$ Department of Gastroenterology, General Hospital of Northern Theater Command (formerly General Hospital of Shenyang Military Area), Shenyang, China; ${ }^{2}$ Graduate School, Jinzhou Medical University, Jinzhou, China; ${ }^{3}$ Department of General Surgery, General Hospital of
\end{abstract} \\ Northern Theater Command (formerly General Hospital of Shenyang Military Area), Shenyang, China; ${ }^{4}$ Medical Library, General Hospital of \\ Northern Theater Command (formerly General Hospital of Shenyang Military Area), Shenyang, China; "These authors contributed equally.
}

\begin{abstract}
Background and objective: In recent years, there has been a significant increase in the incidence of peptic ulcer cases among military personnel. It is important for clinicians to identify the risk factors of peptic ulcer and then implement the appropriate prophylactic measures in a timely manner. This study aims to systematically review the risk factors of peptic ulcer in military personnel.

Methods: We searched literature from the PubMed, EMBASE, Wanfang, China National Knowledge Infrastructure, and VIP databases up to November 17, 2019. Eligible studies analyzed at least one risk factor of peptic ulcer in military personnel with descriptive or comparative data. The risk factors' data were then extracted and tabulated.

Results: Of the 1,008 studies initially identified, 11 were eligible for the present study. The total sample size was 29,925 (ranging from 203 to 10,046). The study population included military officers and soldiers, pilots, armed policemen, and firefighters. The most studied risk factor of peptic ulcer was history of smoking $(n=8)$, followed by high-intensity training $(n=5)$, mental stress $(n=5)$, family history of peptic ulcer $(n=4)$, history of alcohol drinking $(n=4)$, and use of non-steroidal anti-inflammatory drugs $(n=4)$.

Conclusions: Several major risk factors of peptic ulcer have been systematically identified, of which some are modifiable. In the future, proper intervention of these modifiable risk factors may be helpful in preventing military personnel from the development of peptic ulcer.
\end{abstract}

Introduction

Peptic ulcer develops mainly in the stomach or proximal duode-

Keywords: Army; Military; Gastric; Duodenal; Ulcer; Risk factors.

Abbreviations: HP, Helicobacter pylori; NOS, Newcastle-Ottawa scale; NSAIDs, non-steroidal anti-inflammatory drugs; OR, odds ratio.

Received: April 20, 2020; Revised: May 12, 2020; Accepted: May 18, 2020

*Correspondence to: Xingshun Qi, Department of Gastroenterology, General Hospital of Northern Theater Command (formerly General Hospital of Shenyang Military Area), No. 83 Wenhua Road, Shenyang, 110840 Liaoning Province, China. Tel: +86 24-28897603; Fax: +86-24-28851113; E-mail: xingshunqi@126.com; Xiaozhong Guo, Department of Gastroenterology, General Hospital of Northern Theater Command (formerly General Hospital of Shenyang Military Area), No. 83 Wenhua Road, Shenyang, 110840 Liaoning Province, China. Tel: +86-24-28897603; Fax: +86-2428851113; E-mail: guo_xiao_zhong@126.com, guoxiaozhong1962@163.com

How to cite this article: Wang C, Guo X, An Y, Xu S, Zhang D, Qin Y, Qi X. Risk Factors of Peptic Ulcer in Military Personnel: A Systematic Review of the Literature. Exploratory Research and Hypothesis in Medicine 2020;5(3):103-109. doi: 10.14218/ERHM.2020.00026. num. Development of peptic ulcer is primarily due to destruction of the protective mechanisms of the gastrointestinal mucosa, such as the secretion of mucus and bicarbonate, by gastric acid and pep$\sin .{ }^{1}$ The prevalence of peptic ulcer in the general population is $5-10 \%,{ }^{2}$ being an important source of morbidity and mortality worldwide. ${ }^{3}$ Non-steroidal anti-inflammatory drugs (NSAIDs), Helicobacter pylori (HP) infection, and smoking have been identified as major risk factors of peptic ulcer in the general population. ${ }^{4}$

Soldiers and military officers are often under high pressure and in complex environments for a long time, ${ }^{5}$ and it seems that the incidence of peptic ulcer is higher in military personnel than in the general population. ${ }^{6}$ Knowledge regarding the risk factors of peptic ulcer in military personnel is of great significance to guide prevention and treatment of peptic ulcer and improvement in the combat effectiveness of the army. At present, there are only scattered studies on the management of peptic ulcer in military personnel. Herein, we describe our systematic review of the literature to explore the risk factors of peptic ulcer in military personnel. 


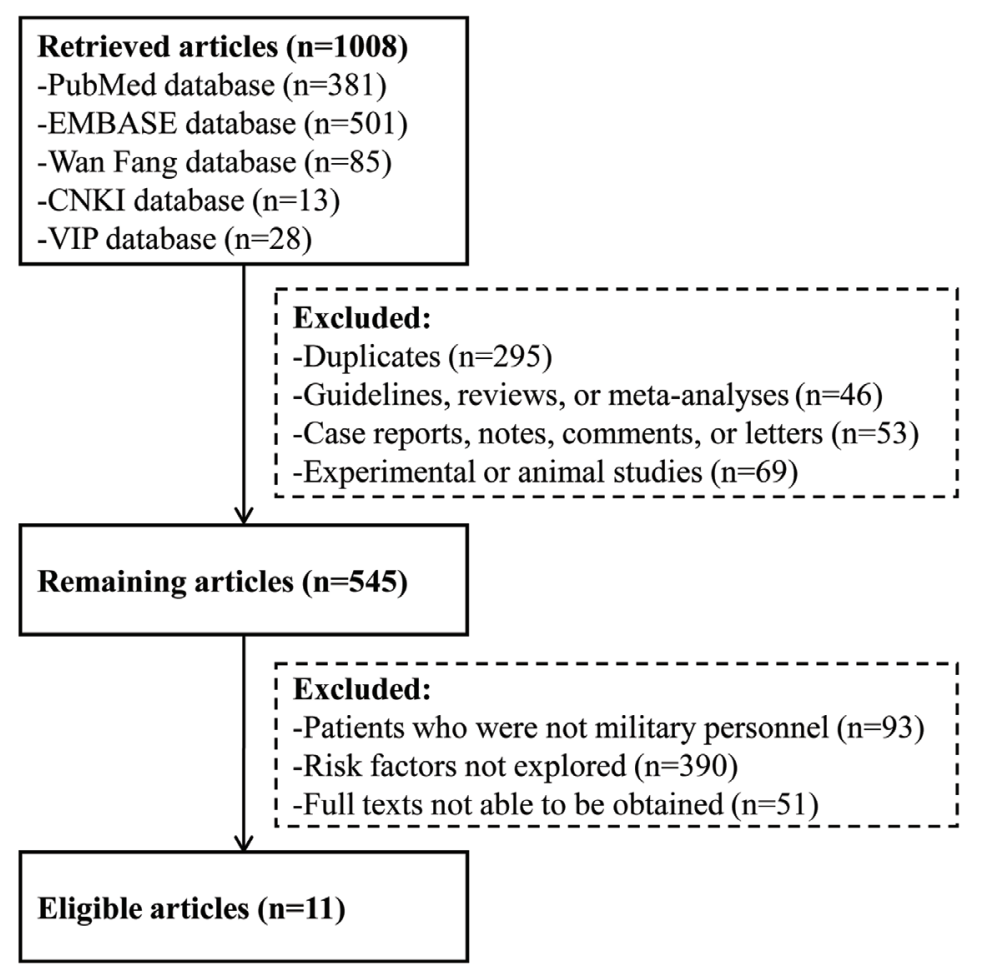

Fig. 1. A flowchart of study inclusion.

\section{Methods}

This systematic review was conducted according to the PRISMA Guidelines. $^{7}$ The PRISMA checklist is shown in the Supplementary Material Table 1.

\section{Registration}

This work was registered in the PROSPERO database.

\section{Search strategy and study selection}

We retrieved all papers via the PubMed, EMBASE, Wanfang, China National Knowledge Infrastructure, and VIP databases. Study publication date, status, and language were not limited, as mentioned by Zhang et al. ${ }^{8}$ The interval was from the earliest available publication until November 17, 2019. A combination of the following keywords was used: ((military) OR (soldier)) AND ((peptic ulcer) OR (gastric ulcer) OR (duodenal ulcer)). The inclusion criteria were as follows: 1) the study participants should be diagnosed with peptic ulcer, including gastric ulcer and/or duodenal ulcer; and 2) the eligible studies should analyze the risk factors of peptic ulcer. Exclusion criteria were as follows: 1) duplicates; 2) case reports, notes, comments, or letters; 3) guidelines, reviews or meta-analyses; 4) experimental or animal studies; 5) patients were not military personnel; 6) risk factors were not explored; and 7) full texts were not able to be obtained.

\section{Data collection}

The following information was extracted from each study: first author; year of publication; study design; enrollment period; total number of military personnel evaluated; incidence of peptic ulcer in military personnel; and risk factors of peptic ulcer.

\section{Study quality assessment}

The quality of the included studies was evaluated using the Newcastle-Ottawa scale (NOS), a widely used tool for assessing the quality of observational/non-randomized studies. ${ }^{9}$ The NOS scale includes the selection of study population, comparability of study groups, and ascertainment of the exposure.

\section{Results}

\section{Characteristics of studies}

We identified 1,008 studies through the PubMed, EMBASE, Wanfang, China National Knowledge Infrastructure, and VIP databases. Finally, 11 studies, which employed endoscopy to diagnose peptic ulcer, were included (Fig. 1). The characteristics of these included studies are listed in Table 1.6,10-19 The included studies were published between 2000 and 2017. The study population mainly included military officers and soldiers, pilots, armed policemen, and firefighters. There were 10 Chinese-language articles and 1 English-language article. Seven studies used random sampling methods to select the study population. Six studies used logistic regression analyses to explore the risk factors of peptic ulcer. The most studied risk factor in all articles was history of smoking, followed by high-intensity training, mental stress, family history of peptic ulcer, history of alcohol drinking, and use of NSAIDs 


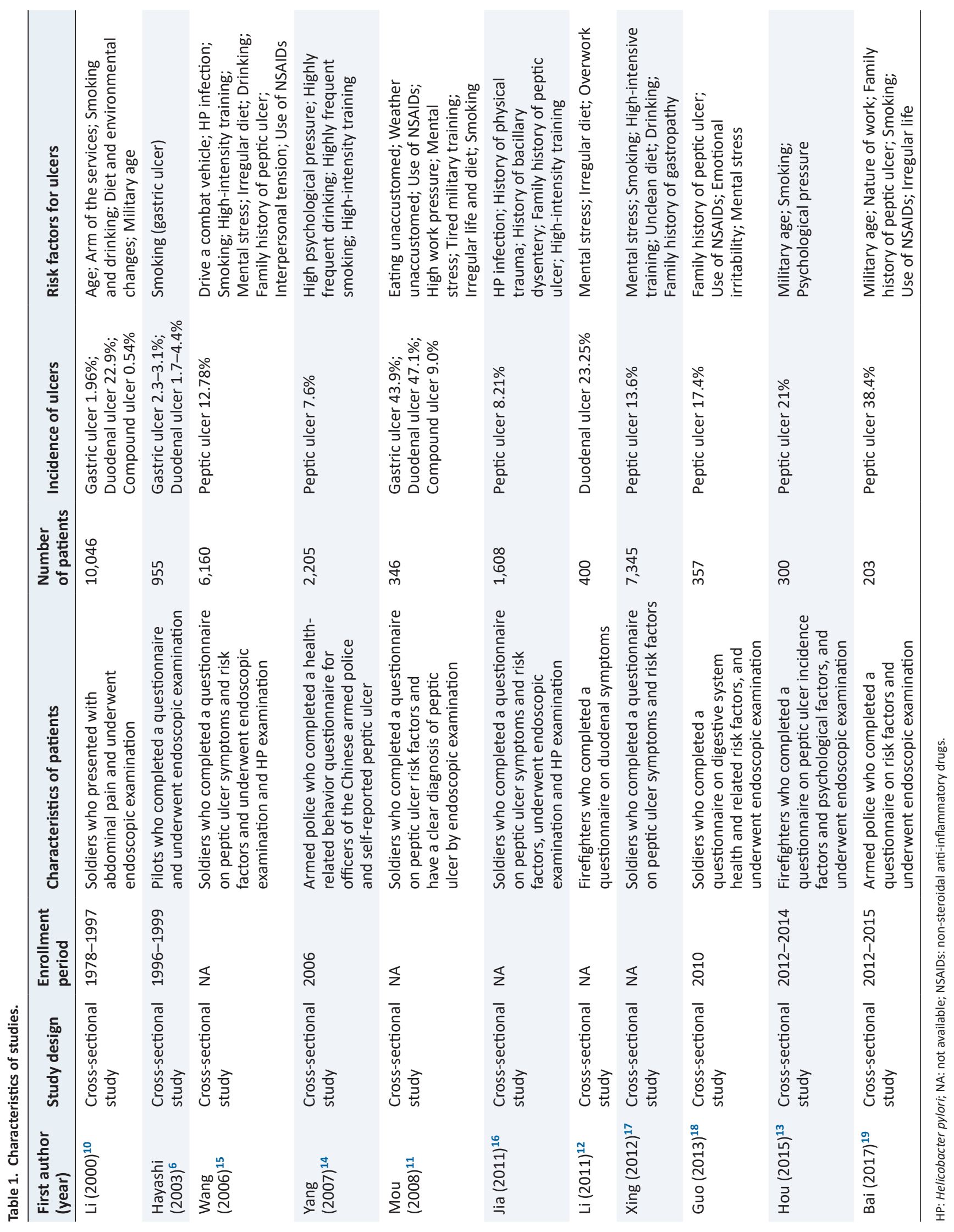


Table 2. Risk factors of peptic ulcers in soldiers and military officers and their frequency.

\begin{tabular}{|c|c|c|}
\hline Risk factors & $\begin{array}{l}\text { Number of papers which ex- } \\
\text { plore such risk factors }\end{array}$ & $\begin{array}{l}\text { Percentage of papers which ex- } \\
\text { plore such risk factors }\end{array}$ \\
\hline History of smoking & 8 & $14.04 \%$ \\
\hline High-intensity training & 5 & $8.77 \%$ \\
\hline Mental stress & 5 & $8.77 \%$ \\
\hline Family history of peptic ulcer & 4 & $7.02 \%$ \\
\hline History of alcohol drinking & 4 & $7.02 \%$ \\
\hline Use of NSAIDs & 4 & $7.02 \%$ \\
\hline Irregular diet & 3 & $5.26 \%$ \\
\hline Military age & 3 & $5.26 \%$ \\
\hline HP infection & 2 & $3.51 \%$ \\
\hline High psychological pressure & 2 & $3.51 \%$ \\
\hline Irregular life & 2 & $3.51 \%$ \\
\hline High work pressure & 1 & $1.75 \%$ \\
\hline Age & 1 & $1.75 \%$ \\
\hline Arm of the services & 1 & $1.75 \%$ \\
\hline Diet and environmental changes & 1 & $1.75 \%$ \\
\hline Drive a combat vehicle & 1 & $1.75 \%$ \\
\hline Eating unaccustomed & 1 & $1.75 \%$ \\
\hline Emotional irritability & 1 & $1.75 \%$ \\
\hline Family history of gastropathy & 1 & $1.75 \%$ \\
\hline History of bacillary dysentery & 1 & $1.75 \%$ \\
\hline History of physical trauma & 1 & $1.75 \%$ \\
\hline Interpersonal tension & 1 & $1.75 \%$ \\
\hline Nature of work & 1 & $1.75 \%$ \\
\hline Overwork & 1 & $1.75 \%$ \\
\hline Unclean diet & 1 & $1.75 \%$ \\
\hline Weather unaccustomed & 1 & $1.75 \%$ \\
\hline
\end{tabular}

NSAIDs: non-steroidal anti-inflammatory drugs.

(Table 2). The quality of these included studies is summarized in Table 3. ${ }^{6,10-19}$ According to the NOS, 4 studies were of moderate to high quality, with a NOS score of $\geq 6$ points, and 7 studies were of low quality.

\section{Risk factors based on descriptive data}

In 2000, Li et al. ${ }^{10}$ performed endoscopy on 10,046 soldiers and military officers who had a complaint of upper abdominal pain. The detection rate of peptic ulcer was $25.40 \%$. They found that the most common locations of duodenal ulcers were the anterior wall and the greater curvature and those of gastric ulcers were the gastric antrum. Peptic ulcer might be related to age, arm of the services, smoking and alcohol drinking, dietary and environmental changes, and military age.

In 2008, Mou et al. ${ }^{11}$ selected 346 soldiers and military officers with a clear endoscopic diagnosis of peptic ulcer as the study population. The patients were divided into recruit training group, daily training group, and field training group. The top three causes of peptic ulcer in the recruit training group were: 1) patients who were tired of military training; 2) patients who were not accustomed to the diet; and 3) patients who did not adapt to the weather. The top three causes of peptic ulcer in the daily training group included: 1) a history of smoking; 2) a history of using NSAIDs; and 3) a high working pressure. The top three causes of peptic ulcer in the field training group included: 1) mental stress; 2) irregular life; and 3) irregular diet.

In 2011, Li et al. ${ }^{12}$ used a cluster random sampling method to investigate 400 firefighters from 16 provinces. There were 92 patients with duodenal ulcers, which accounted for $23.25 \%$ of the total study population. The major cause of duodenal ulcers in professional firefighters was mental stress, followed by irregular diet and overwork.

In 2015, Hou et al. ${ }^{13}$ used a multi-stage stratified overall sampling method to select 300 armed police firefighters, and then asked them to fill out a questionnaire and perform endoscopy to diagnose peptic ulcer. The total detection rate of peptic ulcer was 
Table 3. Results of quality assessment using the Newcastle-Ottawa scale for case-control studies.

\begin{tabular}{|c|c|c|c|c|c|c|c|c|c|}
\hline \multirow{2}{*}{ First author (year) } & \multicolumn{4}{|c|}{ Selection } & \multirow{2}{*}{$\begin{array}{l}\text { Comparability } \\
\text { Q5 }\end{array}$} & \multicolumn{3}{|c|}{ Exposure } & \multirow{2}{*}{ Total } \\
\hline & Q1 & Q2 & Q3 & Q4 & & Q6 & Q7 & Q8 & \\
\hline Li $(2000)^{10}$ & $*$ & $*$ & / & / & $/ /$ & $/ /$ & / & / & 2 \\
\hline Hayashi $(2003)^{6}$ & $*$ & / & / & / & // & $* /$ & / & / & 2 \\
\hline Wang $(2006)^{15}$ & $*$ & $*$ & / & / & $/ /$ & $* *$ & $*$ & $*$ & 6 \\
\hline Yang $(2007)^{14}$ & / & $*$ & / & / & // & */ & / & / & 2 \\
\hline Mou $(2008)^{11}$ & $*$ & $*$ & / & / & $/ /$ & $/ /$ & / & / & 2 \\
\hline Jia $(2011)^{16}$ & $*$ & $*$ & / & / & // & $* /$ & $*$ & $*$ & 5 \\
\hline Li $(2011)^{12}$ & $*$ & $*$ & / & / & // & $* /$ & / & / & 3 \\
\hline Xing $(2012)^{17}$ & $*$ & $*$ & I & / & $/ /$ & $* *$ & $*$ & $*$ & 6 \\
\hline Guo $(2013)^{18}$ & $*$ & $*$ & / & $*$ & $* *$ & $* *$ & $*$ & $*$ & 9 \\
\hline Hou $(2015)^{13}$ & $*$ & $*$ & / & / & // & $* /$ & / & / & 3 \\
\hline Bai $(2017)^{19}$ & $*$ & $*$ & / & $*$ & $/ /$ & $* /$ & $*$ & $*$ & 6 \\
\hline
\end{tabular}

Notes: Q1: Is the case definition adequate? Q2: Representativeness of the cases; Q3: Selection of controls; Q4: Definition of controls; Q5: Comparability of cases and controls on the basis of the design or analysis; Q6: Ascertainment of exposure; Q7: Same method of ascertainment for cases and controls; Q8: Non-response rate.

$21 \%$. Among them, 15 patients had gastric ulcers, 45 had duodenal ulcers, and 3 had complex ulcers. They found that military age, smoking, and psychological pressure were closely related to the onset of peptic ulcer.

\section{Risk factors based on comparative data}

In 2003, Hayashi et al. ${ }^{6}$ obtained data from pilots who filled out a questionnaire to explore the relationship of peptic ulcer with smoking and NSAIDs use. Of the 224 smoking pilots, 27 had open gastric ulcer and 59 had any type of gastric ulcer. Of the 329 nonsmoking pilots, 7 had open gastric ulcer and 44 had any type of gastric ulcer. They found a significant association of smoking with each type of gastric ulcer $(p<0.0005)$. However, there was no relationship between smoking and duodenal ulcer. More importantly, none of them took NSAIDs.

\section{Risk factors based on univariate logistic regression analysis}

In 2007, Yang et al. ${ }^{14}$ used a cluster random sampling method to select 2,253 in-service cadres of a certain armed police department as the study population, and then asked them to fill out a questionnaire. The prevalence of self-reported peptic ulcer among the surveyed cadres was 7.6\%. Univariate logistic regression analysis revealed that high psychological pressure (odds ratio [OR]: 2.396), highly frequent drinking (OR: 1.226), highly frequent smoking (OR: 1.119), and high-intensity training (OR: 1.184) were significant risk factors of peptic ulcer.

\section{Risk factors based on multivariate logistic regression analysis}

In 2006, Wang et al. ${ }^{15}$ used a multi-stage stratified cluster random sampling method to select 6,160 soldiers and military officers in a Southern army. They were divided into three groups, according to their answers on a questionnaire about peptic ulcer symptoms and risk factors. Fifty people were taken from each group to undergo endoscopy. Based on the endoscopic findings, 68 of the 150 sol- diers and military officers were diagnosed with peptic ulcer. The multivariate logistic regression analysis found that driving a combat vehicle (OR: 6.0), HP infection (OR: 4.6), history of smoking (OR: 3.8), high-intensity training (OR: 4.3), mental stress (OR: 3.7), irregular diet (OR: 3.2), alcohol drinking (OR: 2.8), family history of peptic ulcer (OR: 2.1), interpersonal tension (OR: 1.8), and use of NSAIDs (OR: 1.2) were risk factors of peptic ulcer among the 150 soldiers and military officers.

In 2011, Jia et al. ${ }^{16}$ also used a multi-stage stratified cluster random sampling method to select 1,608 soldiers and military officers in the Gobi desert. They were divided into three groups, according to their answers on a questionnaire about peptic ulcer symptoms and risk factors. Thirty people were taken from each group to undergo endoscopy. Based on the endoscopic findings, 33 of the 90 soldiers and military officers were diagnosed with peptic ulcer. The multivariate logistic regression analysis found that HP infection (OR: 3.2), history of physical trauma (OR: 1.9), history of bacillary dysentery (OR: 1.9), family history of peptic ulcer (OR: 2.5), and high-intensity training (OR: 2.3 ) had a close relationship with the occurrence of peptic ulcer symptoms among the 90 soldiers and military officers.

In 2012, Xing et al. ${ }^{17}$ used a multi-stage stratified cluster random sampling method to select 7,345 soldiers and military officers in five locations and four arms of services in cold regions. They were divided into three groups, according to their answers on a questionnaire about peptic ulcer symptoms and risk factors. Fifty people were taken from every group to undergo endoscopy. Based on the endoscopic findings, 69 of the 90 soldiers and military officers were diagnosed with peptic ulcer. The multivariate logistic regression analysis found that mental stress (OR: 3.1), history of smoking (OR: 2.8), high-intensity training (OR: 3.5), unclean diet (OR: 4.6), history of alcohol drinking (OR: 2.1), and family history of gastropathy (OR: 3.4$)$ were closely related to peptic ulcer symptoms among the 90 soldiers and military officers.

In 2013, Guo et al. ${ }^{18}$ used a stratified cluster random sampling method to select 357 soldiers and military officers, and then asked them to fill out a questionnaire and undergo an endoscopic examination. According to the endoscopic findings, patients were divided into a peptic ulcer group and a control group. The mul- 
tivariate logistic regression analysis found that family history of peptic ulcer (OR: 3.610), use of NSAIDs (OR: 4.831), emotional irritability (OR: 3.526), and mental stress (OR: 3.317) were risk factors of peptic ulcer.

In 2017, Bai et al. ${ }^{19}$ conducted a questionnaire survey of 78 patients with peptic ulcer and 125 patients with chronic gastritis diagnosed by endoscopy. The multivariate logistic regression analysis found that military age (OR: 3.591), type of work (OR: 2.432), family history of peptic ulcer (OR: 8.604), history of smoking (OR: 3.907), use of NSAIDs (OR: 4.772), and irregular life (OR: 7.581) were risk factors of peptic ulcer.

\section{Discussion}

We systematically reviewed the risk factors of peptic ulcer in military personnel. The major risk factors were history of smoking, followed by high-intensity training, mental stress, family history of peptic ulcer, history of alcohol drinking, and use of NSAIDs. Such a list of potential risk factors for peptic ulcer should be taken into account for clinical management of this disease in military personnel.

Smoking is an important health behavior problem that can harm almost all major organs. ${ }^{20}$ The use of tobacco in military personnel adversely affects health, combat readiness and performance levels, and increases health care costs. It was reported that military personnel had a higher rate of smoking than the general population $(24.0 \%$ vs. $21.2 \%) .^{21}$ Therefore, it is necessary to raise the awareness of soldiers through education and counseling and to provide medical support for quitting smoking.

Military training is helpful for soldiers to develop the physical quality and endurance and to complete the combat, peace-keeping, and relief missions. ${ }^{22}$ However, sustained intensive training may cause peptic ulcer in soldiers. Therefore, in the future, total training load, nutrition, and recovery should be usually individualized to optimize training adaptation and reduce training-related illness and overtraining. ${ }^{23}$

Family history has been identified as a major risk factor of peptic ulcer. ${ }^{24}$ Due to the biological diversity of the general population, the susceptibility of different individuals to this disease may vary greatly. Additionally, peptic ulcer has obvious familial aggregation, which could be associated with common environmental and genetic factors. ${ }^{25}$

Massive alcohol drinking can cause gastric mucosal inflammation, erosion, and even ulcer. ${ }^{26}$ Alcohol could also delay gastric emptying, interfere with gastroesophageal sphincter activity, stimulate gastric secretion, and damage gastric mucosa, especially in combination with aspirin. ${ }^{27}$ Avoiding alcohol abuse could reduce the incidence of peptic ulcer.

Both HP infection and NSAIDs independently increase the risk for development of peptic ulcer disease. ${ }^{28} \mathrm{HP}$, a Gram-negative bacterium, is the main human pathogen causing chronic progressive gastric mucosal damage. ${ }^{29} \mathrm{HP}$ infection is caused by an imbalance between bacterial virulence factors, host factors, and environmental factors. ${ }^{30}$ NSAIDs have analgesic, anti-inflammation, and antipyretic effects, facilitated by their inhibition of the cyclooxygenase enzyme that synthesizes prostaglandins and thromboxane, thereby leading to damage of gastric and duodenal mucosa. ${ }^{31,32}$ Soldiers are often trained with great intensity and more vulnerable to injury and physical pain, which greatly increases the chance of NSAIDs medication. Therefore, eradication of HP infection and exemption from NSAIDs use can potentially reduce the incidence and severity of peptic ulcer in military personnel.
Our study has some limitations. First, the majority of papers analyzed were from China. Second, some of these evaluated risk factors were analyzed in only one study. Third, the interactions between risk factors were not clearly analyzed. Fourth, the quality of these included studies was not satisfying. Fifth, only a small subset of standardized population was selected. Sixth, some of the study populations were highly selective and the processes of case confirmation were largely inconsistent among these included studies. Seventh, 51 articles, which were published from 1946 to1990, had to be excluded, because their full texts could not be accessed.

\section{Conclusion}

History of smoking, high-intensity training, mental stress, family history of peptic ulcer, history of alcohol drinking, and use of NSAIDs were common risk factors of peptic ulcer in military personnel. Comprehensive identification and early intervention of these risk factors are needed to reduce the incidence of peptic ulcer in military personnel. However, considering that most of the included studies were of poor quality and conducted in very heterogeneous populations, more well-designed and large-scale studies are needed.

\section{Future directions}

In the future, large-scale population-based studies are needed to validate the effect of these risk factors on peptic ulcer in military personnel. An integration of these risk factors into a predictive model will be valuable to evaluate the risk of peptic ulcer. Close endoscopic screening on high-risk patients and proper interventions of these modifiable risk factors should be considered to improve these patients' outcomes.

\section{Supporting information}

Supplementary material for this article is available at https://doi. org/10.14218/ERHM.2020.00026.

\section{Supplementary Table 1. PRISMA 2009 Checklist.}

\section{Acknowledgments}

None.

\section{Data sharing statement}

No additional data are available.

Funding

There is no funding related to this work.

\section{Conflict of interest}

None. 
Author contributions

Conceptualization (XQ), methodology (CW, XQ), validation (XQ, $\mathrm{XG}$ ), formal analysis (CW, XG, YA, SX, DZ, YQ, XQ), investigation (CW, XG, YA, SX, DZ, XQ), data curation (CW, XQ), writing of the original draft $(\mathrm{CW}, \mathrm{XQ})$, writing, review and editing of the manuscript (CW, XG, YA, SX, DZ, YQ, XQ), supervision of the project $(\mathrm{XG}, \mathrm{XQ})$, administration of the project $(\mathrm{XQ}, \mathrm{XG})$. All authors have made an intellectual contribution to the manuscript and approved the submission.

\section{References}

[1] Sung JJ, Kuipers EJ, El-Serag HB. Systematic review: the global incidence and prevalence of peptic ulcer disease. Aliment Pharmacol Ther 2009;29(9):938-946. doi:10.1111/j.1365-2036.2009.03960.x.

[2] Lanas A, Chan FKL. Peptic ulcer disease. Lancet 2017;390(10094):613624. doi:10.1016/s0140-6736(16)32404-7.

[3] Kavitt RT, Lipowska AM, Anyane-Yeboa A, Gralnek IM. Diagnosis and Treatment of Peptic Ulcer Disease. Am J Med 2019;132(4):447-456. doi:10.1016/j.amjmed.2018.12.009.

[4] Albaqawi ASB, El-Fetoh NMA, Alanazi RFA, Alanazi NSF, Alrayya SE, Alanazi ANM, et al. Profile of peptic ulcer disease and its risk factors in Arar, Northern Saudi Arabia. Electron Physician 2017;9(11):57405745. doi:10.19082/5740.

[5] Chou HW, Tzeng WC, Chou YC, Yeh HW, Chang HA, Kao YC, et al. Stress, Sleep and Depressive Symptoms in Active Duty Military Personnel. Am J Med Sci 2016;352(2):146-153. doi:10.1016/j.amjms.2016.05.013.

[6] Hayashi T, Ohrui N, Kobayashi M, Ohashi K, Ikeda T. Peptic ulcer data from 4 years of endoscopy in 955 pilots of the Japan Air Self Defense Force. Aviat Space Environ Med 2003;74(10):1067-1071.

[7] Moher D, Liberati A, Tetzlaff J, Altman DG, PRISMA Group. Preferred reporting items for systematic reviews and meta-analyses: the PRISMA statement. BMJ 2009;339:b2535. doi:10.1136/bmj.b2535.

[8] Zhang L, Gerson L, Maluf-Filho F. Systematic review and meta-analysis in Gl endoscopy: Why do we need them? How can we read them? Should we trust them? Gastrointest Endosc 2018;88(1):139-150. doi:10.1016/j.gie.2018.03.001.

[9] Stang A. Critical evaluation of the Newcastle-Ottawa scale for the assessment of the quality of nonrandomized studies in meta-analyses. Eur J Epidemiol 2010;25(9):603-5. doi:10.1007/s10654-010-9491-z.

[10] Li P, Xue HX, Zhou GH, Leng MF, Yao XN, Gao HW. Clinical Epidemiological Investigation on Peptic Ulcer in Southern Troops (in Chinese). Clin J Med Off 2000;28(1):44-45.

[11] Mou WM, Zhou SJ, Ren CW, Liu CL. Risk factors of peptic ulcer in soldiers and prevention of recurrence by intermittent oral administration of ranitidine (in Chinese). Shengyang Budui Yiyao 2008;21(1):57-59.

[12] Li X. The etiology of multiple duodenal ulcerations in professional firefighters was discussed from the perspective of TCM syndrome differentiation (in Chinese). Medical Information 2011;24(5):178-179.

[13] Hou L. Analysis on factors of fire fighters' high incidence of peptic ulcer (in Chinese). For all Health 2015;9(11):18.

[14] Yang T, Ji Y, Li YF, Zhu ZL, Cao CX, Li CH, et al. The status of health related behaviors survey among officers in Chinese people's armed police forces (in Chinese). Chin J Behav Med Sci 2007;16(11):1016-1018.

[15] Wang W, Wang XJ, Wang R, Li DZ, Wang YB, Zhang ZJ, et al. Prevalence and related factors of peptic ulcer in military personnel of China (in Chinese). Medical Journal of Chinese People's Liberation Army 2006;31(9):869-872.

[16] Jia SY, Jia HY, Yin LJ, Yang CN, Guo XY, Wang AH, et al. Prevalence and related factors of peptic ulcer among military personnel in the Gobi desert (in Chinese). Med J Natl Defending Forces Northwest China 2011;32(4):244-246.

[17] Xing AH, Li DL, Shao K, Guo ZY, Yan TC, Wang YY, et al. Epidemiological investigation on traditional chinese medicine syndrome of peptic ulcer in military personnel in cold region (in Chinese). Journal of Preventive Medicine of Chinese People's Liberation Army 2012;30(1):13-16.

[18] Guo ZY, Li DL, Wang YY. Investigation on risk factors of peptic ulcer among soldiers in the northeast war zone (in Chinese). Occupation and Health 2013;29(10):1248-1250.

[19] Bai X, Zhang R, Lei XH. Analysis of factors associated with peptic ulcer in Chinese People's Armed Police Force (in Chinese). Medical Journal of the Chinese People's Armed Police Force 2017;28(1):19-21. doi: doi:10.3969/j.issn.1004-3594.2017.01.006.

[20] Brown JM, Anderson Goodell EM, Williams J, Bray RM. Socioecological Risk and Protective Factors for Smoking Among Active Duty U.S. Military Personnel. Mil Med 2018;183(7-8):e231-e239. doi:10.1093/ milmed/usx021.

[21] Ulanday KT, Jeffery DD, Nebeling L, Srinivasan S. Perceived Deterrence of Cigarette Use and Smoking Status Among Active Duty Military Personnel. Mil Med 2017;182(5):e1733-e1741. doi:10.7205/ milmed-d-16-00201.

[22] Grant CC, Mongwe L, Janse van Rensburg DC, Fletcher L, Wood PS, Terblanche E, et al. The Difference Between Exercise-Induced Autonomic and Fitness Changes Measured After 12 and 20 Weeks of Medium-to-High Intensity Military Training. J Strength Cond Res 2016;30(9):2453-2459. doi:10.1519/JSC.0b013e3182a1fe46.

[23] Kyröläinen H, Pihlainen K, Vaara JP, Ojanen T, Santtila M. Optimising training adaptations and performance in military environment. J Sci Med Sport 2018;21(11):1131-1138. doi:10.1016/j.jsams. 2017.11.019.

[24] Brenner H, Rothenbacher D, Bode G, Adler G. The individual and joint contributions of Helicobacter pylori infection and family history to the risk for peptic ulcer disease. J Infect Dis 1998;177(4):1124-1127. doi:10.1086/517410.

[25] Tarpila S, Samloff IM, Pikkarainen P, Vuoristo M, Ihamäki T. Endoscopic and clinical findings in first-degree relative of duodenal ulcer patients and control subjects. Scand J Gastroenterol 1982;17(4):503506. doi:10.3109/00365528209182239.

[26] Selmi S, Rtibi K, Grami D, Sebai H, Marzouki L. Protective effects of orange (Citrus sinensis L.) peel aqueous extract and hesperidin on oxidative stress and peptic ulcer induced by alcohol in rat. Lipids Health Dis 2017;16(1):152. doi:10.1186/s12944-017-0546-y.

[27] Chou SP. An examination of the alcohol consumption and peptic ulcer association-results of a national survey. Alcohol Clin Exp Res 1994;18(1):149-153. doi:10.1111/j.1530-0277.1994.tb00895.x.

[28] Sostres C, Carrera-Lasfuentes P, Benito R, Roncales P, Arruebo M, Arroyo $\mathrm{MT}$, et al. Peptic Ulcer Bleeding Risk. The Role of Helicobacter Pylori Infection in NSAID/Low-Dose Aspirin Users. Am J Gastroenterol 2015;110(5):684-689. doi:10.1038/ajg.2015.98.

[29] Lahner E, Carabotti M, Annibale B. Treatment of Helicobacter pylori infection in atrophic gastritis. World J Gastroenterol 2018;24(22):2373-2380. doi:10.3748/wjg.v24.i22.2373.

[30] Yeo SH, Yang CH. Peptic Ulcer Disease Associated with Helicobacter pylori Infection (in Korean). Korean J Gastroenterol 2016;67(6):289299. doi:10.4166/kjg.2016.67.6.289.

[31] Shim YK, Kim N. Nonsteroidal Anti-inflammatory Drug and Aspirininduced Peptic Ulcer Disease (in Korean). Korean J Gastroenterol 2016;67(6):300-312. doi:10.4166/kjg.2016.67.6.300.

[32] Melcarne L, Garcia-Iglesias P, Calvet X. Management of NSAIDassociated peptic ulcer disease. Expert Rev Gastroenterol Hepatol 2016;10(6):723-733. doi:10.1586/17474124.2016.1142872. 